\title{
Estimation of vapour pressure and partial pressure of subliming compounds by low-pressure thermogravimetry
}

\author{
G V KUNTE*, UJWALA AIL ${ }^{\dagger}$, P K AJIKUMAR ${ }^{\dagger}$, A K TYAGI', \\ $S$ A SHIVASHANKAR and A M UMARJI \\ Materials Research Centre, Indian Institute of Science, Bangalore 560 012, India \\ ${ }^{\dagger}$ Materials Science Division, Indira Gandhi Centre for Atomic Research, Kalpakkam 603 102, India
}

MS received 1 August 2009

\begin{abstract}
A method for the estimation of vapour pressure and partial pressure of subliming compounds under reduced pressure, using rising temperature thermogravimetry, is described in this paper. The method is based on our recently developed procedure to estimate the vapour pressure from ambient pressure thermogravimetric data using Langmuir equation. Using benzoic acid as the calibration standard, vapour pressuretemperature curves are calculated at 80,160 and $1000 \mathrm{mbar}$ for salicylic acid and vanadyl bis-2,4pentanedionate, a precursor used for chemical vapour deposition of vanadium oxides. Using a modification of the Langmuir equation, the partial pressure of these materials at different total pressures is also determined as a function of temperature. Such data can be useful for the deposition of multi-metal oxide thin films or doped thin films by chemical vapour deposition (CVD).
\end{abstract}

Keywords. Low-pressure thermogravimetry; vapour pressure; Langmuir equation; partial pressure; precursor; metalorganic chemical vapour deposition.

\section{Introduction}

The determination of vapour pressure of volatile substances and subliming solids is important because of the use of such data in various applications. Vapour pressure data are very important for industrial process control, in manufacturing processes and in estimating exposure limits to volatile substances and the shelf-lives of volatile compounds. Another important application of such data is in the study of precursors for thin-film deposition, especially by metalorganic chemical vapour deposition (MOCVD). Vapour pressure data for the precursors used in CVD are often not readily available in the literature or in databases.

Furthermore, it is important to know the partial pressure of a precursor used for MOCVD under the conditions of deposition. Partial pressure data are essential for a quantitative understanding of the reaction processes involved in the deposition process and hence for the optimization of the deposition conditions. Such data also help predict the products of the deposition process.

Various methods are used for the determination of vapour pressure. The most important among them are effusion using a Knudsen cell (Ribeiro Da Silva et al 1990), boiling point determination under reduced pressures (Goodrum and Siesel 1996) and isothermal ther-

\footnotetext{
*Author for correspondence (girish.kunte@ gmail.com)
}

mogravimetry (Gueckel et al 1974). Rising temperature thermogravimetry was used recently for the determination of vapour pressure based on the Langmuir equation (Price and Hawkins 1998). Rising temperature thermogravimetry has been used for the determination of the vapour pressure of pharmaceuticals (Chatterjee et al 2002), dyes and other industrially important organic substances (Gueckel et al 1974; Phang and Dollimore 2001). We have shown recently the usefulness of rising temperature thermogravimetric data in the calculation of the vapour pressure of precursors used in MOCVD (Kunte et al 2008).

Recently, a modified approach to the Langmuir equation was reported wherein the mass diffusion of the subliming substance due to the flowing gas in the thermogravimetric analysis (TGA) apparatus was taken into account. This has been shown to be necessary because the Langmuir equation is strictly valid only for vapourization into absolute vacuum (Pieterse and Focke 2003). In the presence of ambient gases, molecular collisions between the evaporating molecules and the atmospheric molecules affect the vapour pressure of the vaporizing entity. Nevertheless, the validity of using the Langmuir equation has been established by showing that the vapour pressure estimated by using the Langmuir equation matches closely with that measured by the Knudsen method under appropriate conditions and with the appropriate choice of a standard (Price and Hawkins 1998). This method is well accepted in the literature as an accurate method for determining vapour pressure. 
In this paper, we demonstrate the use of low-pressure thermogravimetry in the calculation of the vapour pressure of a material and its partial pressure, under any given conditions of pressure, as a function of temperature. The partial pressure as a function of temperature is calculated using a modified form of the Langmuir equation for salicylic acid and for vanadyl bis-2,4-pentanedionate, a precursor used for the MOCVD of vanadium oxides (Sahana et al 2002). Using the partial pressure data, the amount of metal in the vapour is estimated.

\section{Principle of the measurement}

The principle behind the estimation of vapour pressure using thermogravimetry (TG) is the Langmuir equation (Langmuir 1913)

$$
P=\left[\left(\frac{\mathrm{d} m}{\mathrm{~d} t}\right) \sqrt{\frac{T}{M}}\right]\left[\frac{\sqrt{2 \pi R}}{\alpha}\right],
$$

where $P$ is the vapour pressure at temperature $T, \mathrm{~d} m / \mathrm{d} t$ the rate of weight loss per unit surface area during the TG experiment, $M$ the molecular mass of the material, $R$ the gas constant and $\alpha$ the vaporization coefficient. In vacuum, $\alpha$ is equal to 1 , but in the flowing gas environment of the TG experiment, $\alpha$ is significantly different and has to be determined experimentally (Langmuir 1913).

The value of $\alpha$ is determined for a particular set of experimental parameters, on a particular instrument, using a calibration standard. The value of $\alpha$ is determined from the Langmuir equation using the thermogravimetry data for a standard whose vapour pressure is known. This value of $\alpha$ is used to calculate the vapour pressuretemperature data of other compounds using the Langmuir equation.

In the Langmuir equation, the vaporization coefficient $\alpha$ correlates the rate of mass loss of the material under the experimental conditions with the vapour pressure. Hence, if the term $\alpha$ is not used in the equation, the rate of mass loss corresponds to the partial pressure of the material under the conditions of the experiment, which is not equal to the vapour pressure, but is lower, due to recondensation of the material as it evaporates. Thus, by omitting the vaporization coefficient from (1), we obtain the (2), which may be referred to as the modified Langmuir equation

$$
P=\left[\left(\frac{\mathrm{d} m}{\mathrm{~d} t}\right) \sqrt{\frac{T}{M}}\right](\sqrt{2 \pi R}) .
$$

In this equation, the term $\mathrm{d} m / \mathrm{d} t$ thus relates to the partial pressure of the material under the conditions of the TG experiment. The modified equation may thus be used to estimate the partial pressure of a material under any given conditions of (total) pressure by using thermogravimetry under those conditions.
Further, from the partial pressure $P$, it is possible to determine the number of moles of material in the vapour phase using the ideal gas equation, $P V=n R T$, where $P$ is the partial pressure, $V$ the volume, $n$ number of moles (of the vapour), $R$ the universal gas constant and $T$ the temperature.

\section{Experimental}

Low-pressure thermogravimetric measurements were carried out on a Setaram SETSYS 16/18 simultaneous TG/DTA (thermogravimetric/differential thermal analyser). A heating rate of $10^{\circ} \mathrm{C} / \mathrm{min}$ and nitrogen gas flow of $100 \mathrm{sccm}$ were used in all the thermogravimetric measurements. Benzoic acid was used as the standard in this work to calculate the vaporization coefficient $\alpha$. The coefficient was determined as described in our previous work (Kunte et al 2008). The vapour pressure data for benzoic acid were taken from the literature (Stull 1947). TG analysis was subsequently carried out and vapour pressure-temperature data calculated for salicylic acid (Qualigens, 98\%, AR-grade) and vanadyl bis-2,4-pentanedionate, a precursor for the MOCVD of vanadium oxides. The complex vanadyl-2,4-pentanedionate was prepared and characterized using a method described in the literature (Woollins 2003). Low-pressure thermogravimetry measurements were carried out at 160 and $80 \mathrm{mbar}$, and the partial pressures of the two compounds were calculated as a function of temperature at these pressures using the modified Langmuir equation. Using the partial pressure data and the ideal gas equation, the metal content in the vapour phase was estimated.

\section{Results and discussion}

\subsection{Determination of the vaporization coefficient $\alpha$}

The thermogramimetric plots for benzoic acid at 1000 , 160 and 80 mbar are shown in figure 1. It is observed that, at lower pressures, the weight loss curve shifts to a lower temperature. The vapour pressure data for benzoic acid were taken from the literature (Stull 1947) and, using the Langmuir equation (1), the values of the vaporization coefficient $\alpha$ under the three operating conditions were calculated. The values are listed in table 1 . By using the appropriate value of $\alpha$, calculated from the benzoic acid data obtained under a certain set of thermogravimetric conditions, the vapour pressure of the test material can be calculated from the data obtained under those operating conditions. The values of $\alpha$ were calculated from the TGA data of benzoic acid obtained at the corresponding pressures. It can be seen that as the pressure reduces, the value of $\alpha$ increases. It is expected, according to the Langmuir equation, that at absolute vacuum, the value of $\alpha$ is unity. The observed values are consistent with this, 
and as the pressure is reduced, the value of $\alpha$ approaches unity.

\subsection{Determination of vapour pressure and partial pressure of salicylic acid}

The vapour pressure curves for salicylic acid were calculated from the thermogravimetry measurements carried out at three different pressures, i.e. 1000, 160 and 80 mbar. The results are shown in figure 2 . It can be seen that there is a very good match between the values of vapour pressure obtained from the three different experiments conducted under three different conditions. The data from the literature (Stull 1947) are also plotted in the figure. A very good agreement between the data obtained from TG at different pressures and standard data from the literature is clearly visible. This shows that by using the appropriate $\alpha$ the vapour pressure can be obtained from the TG data recorded at any pressure, provided the value of $\alpha$ at that pressure is known.

Without using the vaporization coefficient $\alpha$, but using the modified Langmuir equation (2), the partial pressure of the material was calculated as a function of temperature at three different pressures. The partial pressure-temperature curves at three different pressures, 1000, 160 and 80 mbar for salicylic acid, obtained in this manner, are shown in figure 3 . It is observed from this graph that as the total

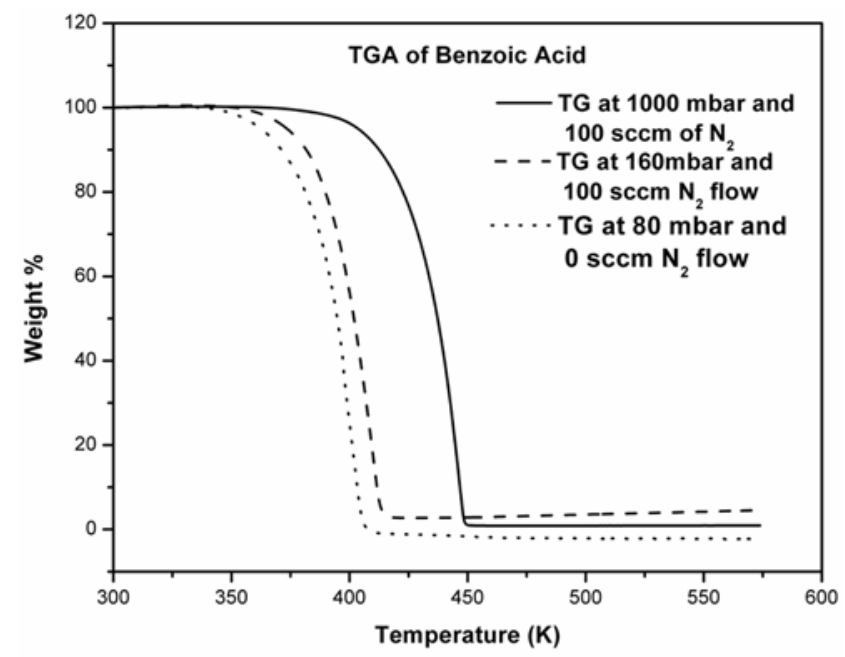

Figure 1. TGA plots for benzoic acid at 80, 160 and 1000 mbar.

Table 1. Table showing values of $\alpha$ as a function of pressure.

\begin{tabular}{rc}
\hline Pressure (mbar) & $\alpha$ \\
\hline 1000 & $1.949 \times 10^{-4}$ \\
160 & $1.188 \times 10^{-3}$ \\
80 & $2.238 \times 10^{-3}$ \\
\hline
\end{tabular}

pressure is reduced, the partial pressure of the material is higher, at the same temperature.

\subsection{Determination of vapour pressure and partial pressure of a vanadium precursor for MOCVD}

The TG/DTG (differential thermogravimetry) plot for vanadyl bis-2,4-pentanedionate is shown in figure 4 . The TG shows a single-step weight loss, corresponding to sublimation of the complex. The DTG shows a monotonic rise followed by a sharp fall, indicating that the process is of the zeroth order. These two trends indicate that the complex might be a good precursor for CVD applications.

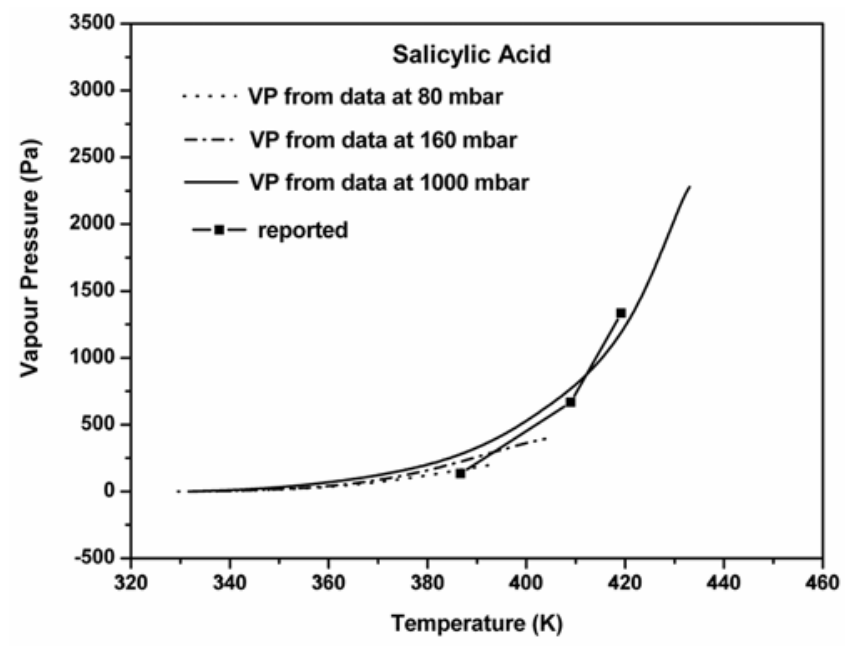

Figure 2. Vapour pressure-temperature plot for salicylic acid from low-pressure thermogravimetry data obtained at 80,160 and 1000 mbar.

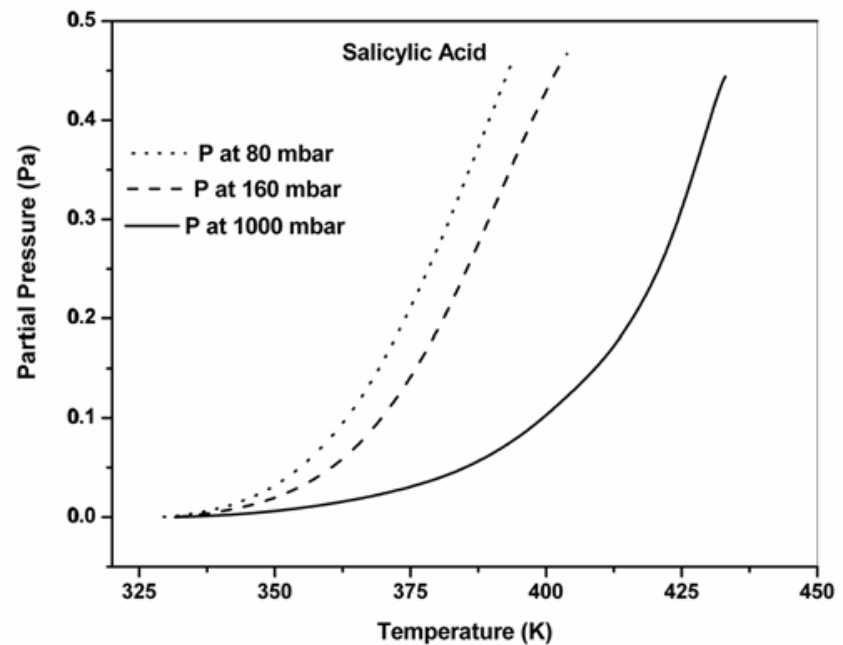

Figure 3. Partial pressure-temperature plot for salicylic acid at different pressures. 


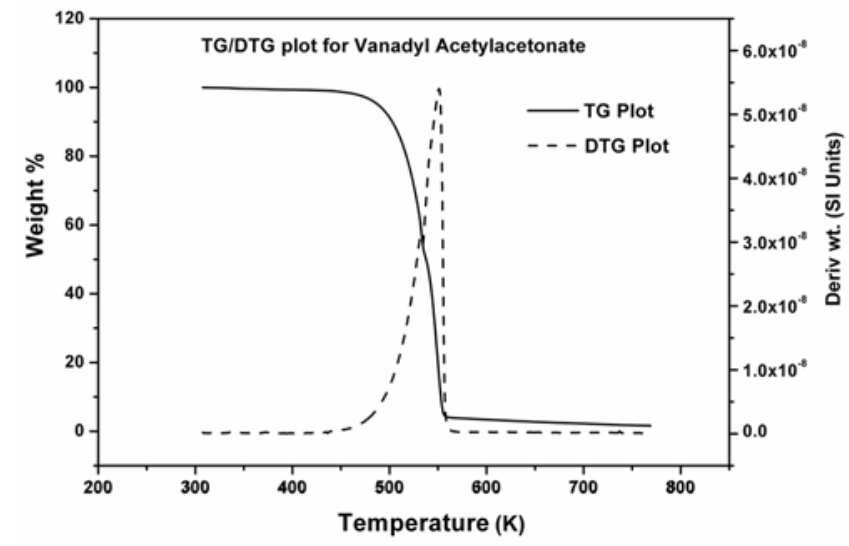

Figure 4. TG/DTG plot of vanadyl bis-2,4-pentanedionate.

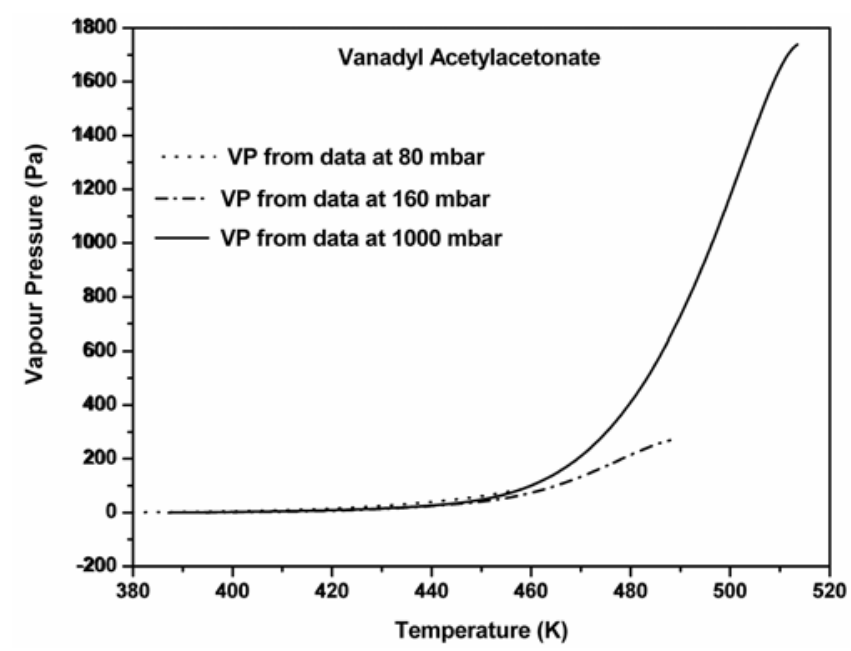

Figure 5. Vapour pressure-temperature plot for vanadyl bis2,4-pentanedionate from low-pressure thermogravimetry data obtained at 80,160 and 1000 mbar.

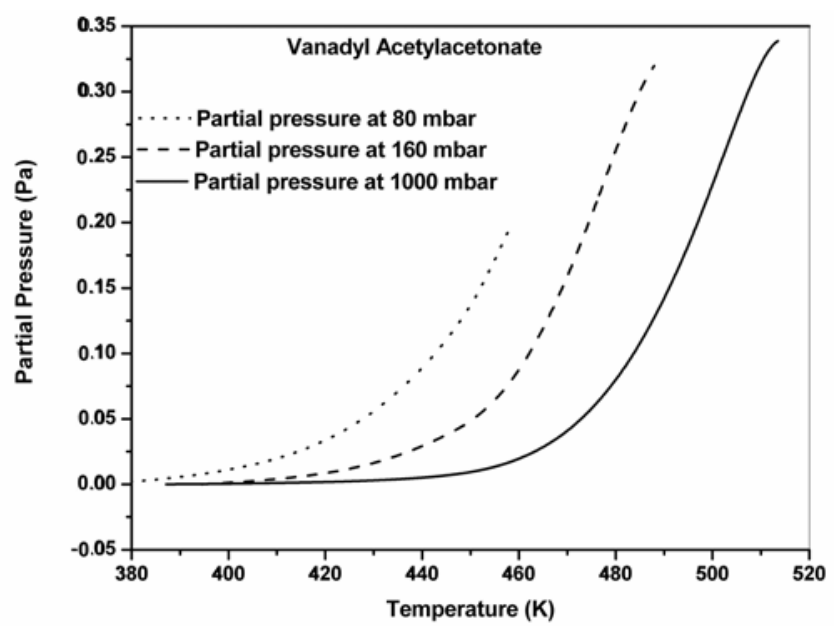

Figure 6. Partial pressure-temperature plot for vanadyl bis2,4-pentanedionate at different pressures.

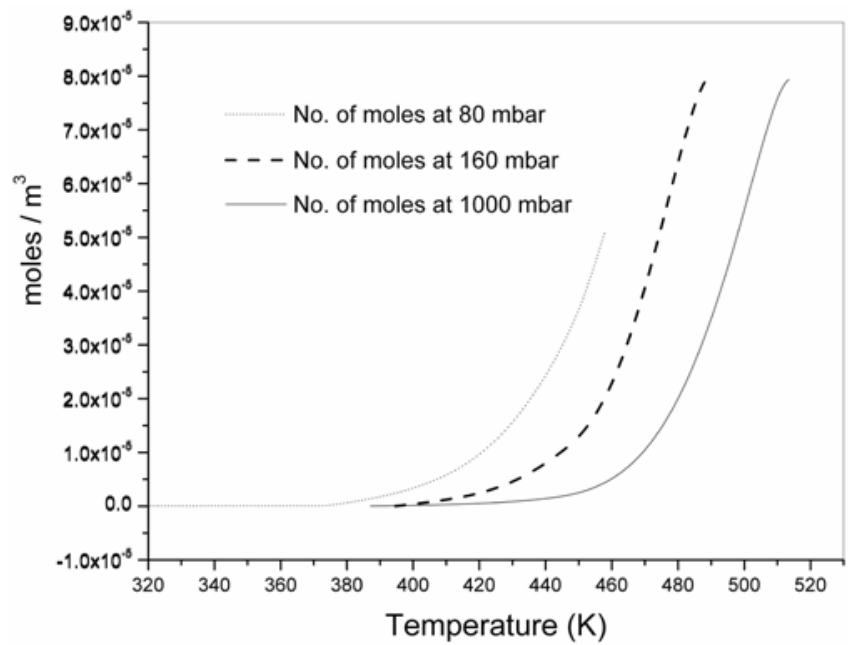

Figure 7. Molar concentration of vanadium metal as a function of temperature at different pressures.

The vapour pressure of vanadyl bis-2,4-pentanedionate was calculated from thermogravimetric measurements made at three different pressures. The results are shown in the figure 5. There is very good consistency observed between the vapour pressure data obtained from the three experiments.

In a similar manner, the partial pressure as a function of temperature of vanadyl bis-2,4-pentanedionate was calculated at the three different pressures, i.e. 80,160 and 1000 mbar. The results are shown in figure 6. Thus, using the modified Langmuir equation, it is possible to estimate directly the partial pressure of a material, under a certain set of conditions, from the low-pressure thermogravimetric data obtained under those conditions. Further, using the partial pressure at a given temperature, and the ideal gas equation, the number of moles in the vapour phase was determined. Figure 7 shows a plot of the vanadium metal concentration as a function of temperature at different pressures. This quantitative estimation of metal content in the vapour phase can be very important to the optimization of process parameters for CVD and can help in predicting the products of the CVD process. These data can be particularly useful in the deposition of multi-metal oxide thin films or doped thin films prepared by CVD.

In low-pressure MOCVD processes using subliming solid precursors, such as the vanadium complex, the precursor vaporization chamber is often connected to the pump via the CVD chamber. This reduces the total pressure in the vaporizer, though it is often higher than in the CVD chamber. Thus, the partial pressure data obtained from low-pressure thermogravimetric measurements are practically relevant and useful.

\section{Conclusions}

A technique for using low-pressure thermogravimetry for the estimation of vapour pressure and partial pressure of 
subliming solids as a function of temperature, based on the Langmuir equation, has been described in this work. These methods have been used to calculate the vapour pressure and partial pressure at different pressures for vanadyl acetylacetonate, a precursor used for the MOCVD of vanadium oxides, is calculated. From the partial pressure data, the metal content in the vapour phase is estimated. This demonstrates the applicability of the present technique to the estimation of the vapour pressure and the partial pressures of subliming precursors for MOCVD. Such data, often not readily available, are important to detailed studies of MOCVD processes.

\section{References}

Chatterjee K, Hazra A, Dollimore D and Alexander K S 2002 Eur. J. Pharm. Biopharmaceutics 54171
Goodrum J W and Siesel FM 1996 J. Therm. Anal. 44 1251

Gueckel W, Rittig F R and Symatschke G 1974 Pestic. Sci. 5 393

Kunte G V, Shivashankar S A and Umarji A M 2008 Meas. Sci. Technol. 19571

Langmuir I 1913 Phys. Rev. 2329

Phang P and Dollimore D 2001 Thermochim. Acta 367-368 263

Pieterse N and Focke W W 2003 Therchim. Acta 406 191

Price D M and Hawkins M 1998 Thermochim. Acta 31519

Ribeiro Da Silva M A V and Monte M K S 1990 Thermochim. Acta 174169

Sahana M B, Dharmaprakash M S and Shivashankar S A 2002 J. Mater. Chem. 12333

Stull D R 1947 Industr. Eng. Chem. 39517

Woollins D J (ed.) 2003 Inorganic experiments (Weinheim: Wiley-VCH) 